\title{
Analyses of Chloroplast Genomic and Morphological Evolutionomy of Yulania Subsect. Cylindricae (Magnoliaceae)
}

\author{
Da-Li Fu ${ }^{1,4, ~ *, ~ H a o ~ F u ², ~ Y u e ~ Q i n ~}{ }^{1,4}$, Dao-Shun Zhou ${ }^{1,4}$, Run-Mei Duan ${ }^{3}$ \\ ${ }^{1}$ Non-timber Forestry Research and Development Center, Chinese Academy of Forestry, Zhengzhou, China \\ ${ }^{2}$ The General Station of Forest and Grassland Pest Control of National Forestry and Grassland Administration, Shenyang, China \\ ${ }^{3}$ Experimental Center of Tropical Forestry, Chinese Academy of Forestry, Pingxiang, China \\ ${ }^{4}$ Key Laboratory of Non-timber Forest Germplasm Enhancement \& Utilization of National Forestry and Grassland Administration, Zhengzhou, \\ China
}

Email address:

fu_dali@163.com (Da-Li Fu)

${ }^{*}$ Corresponding author

\section{To cite this article:}

Da-Li Fu, Hao Fu, Yue Qin, Dao-Shun Zhou, Run-Mei Duan. Analyses of Chloroplast Genomic and Morphological Evolutionomy of Yulania Subsect. Cylindricae (Magnoliaceae). American Journal of Agriculture and Forestry. Special Issue: The New Evolutionary Theory \& Practice. Vol. 7, No. 5, 2019, pp. 200-211. doi: 10.11648/j.ajaf.20190705.16

Received: August 23, 2019; Accepted: August 28, 2019; Published: September 20, 2019

\begin{abstract}
To scientifically settle the puzzle of origin of fruit plants, the chloroplast genomic sequences of three species of Yulania subsect. Cylindricae (Spongb.) D. L. Fu, subsect. comb. nov. (Magnoliaceae) were determined, which were compared with some taxa by means of the typical algorithm, a new method for genomic evolutionomy based on the evolutionary continuity principle. The results indicated that among some representative species of Gymnospermophyda, Yulania puberula D. L. Fu, sp. nov. has the closest relatively evolutionary relationship with Ginkgo biloba, not with the species of Cycas, Welwitschia or Ephedra, which indicated that fruit plants originated from Ginkgoopsida, not from Cycadopsida thought by the euanthium-theory or Chlamydopsermopsida thought by the pseudoanthium-theory. Among some representative species of Fructophyta, Ginkgo biloba has the closest relatively evolutionary relationship with Yulania puberula indicating that the new species is the relatively most primitive species of fruit plants, which is consistent with the results of morphological evolutionomy. The evolutionary system of Magnoliaceae includes 4 natural genera: Yulania Spach, Magnolia L., Michelia L. and Liriodendron L., whose boundaries all are PHS $(17 \mathrm{bp})=0.93$. Furthermore Yulania subsect. Cylindricae and its three species were described or emended. The holotype of new species of Yulania puberula was designated, whose main typici-evolutionary characters, including diagnostic differences and particularities, was given and illustrated. The epitype of Y. shizhenii was designated and four synonyms of Y. cylindrica were listed. Typical algorithm is a scientific method of genomic evolutionomy and a scientifically new tool to solve the puzzle of evolutionomy of fruit plants.
\end{abstract}

Keywords: Typical Algorithm, Chloroplast Complete Genome, Evolutionomy, Yulania Puberula, Yulania Subsect. Cylindricae, Magnoliaceae, Fructophyta, New Species

\section{Introduction}

Fructophyta D. L. Fu \& H. Fu [1], a new division built in 2018 , including all fruit or flowering plants, commonly called angiosperms, occupy the highest evolutionary phylum taxa and an important position in terrestrial ecosystems and human wellbeing. The origin and evolution of fruit plants remain a mystery, as that the British plant taxonomists P. H. Davis and V. H. Heywood pointed out in their famous book Principles of Angiosperm Taxonomy, the origin and phylogeny of angiosperms have puzzled plant systematics for quite some time. The famous botanist, Professor Zhang Hongda [2] also 
pointed out that: "because of the long age, it is rare to find the complete fossils of original flowering plants belonging to the Triassic.... at present, that we do not have a rational evolutionary system of flowering plant is understandable."

There are two opposite theories about the origin of fruit plants: euanthium-theory and pseudoanthium-theory. The representative systems of pseudoanthium-theory were Engler system [3] and H. D. Zhang system [2], which thought flowering plants originated from Chlamydopsermopsida of Gymnospermophyta. Casuarinaceae R. Br. are the most primitive taxa in Engler system [3] and Trochodendraceae Prant. are the most primitive taxa in H. D. Zhang system [2]. The representative systems of euanthium-theory were Hutchinson system [4], Takhtajan system [5], Cronquist system [6] and C. Y. Wu System [7], which thought flowering plants originated from Cycadopsida of Gymnospermophyta. Winteraceae Lindley are the most primitive taxa of flowering plants in Takhtajan system [5] and Cronquist system [6], and Magnoliaceae Juss. are the most primitive taxa of flowering plants in Hutchinson system [4] and C. Y. Wu system [7].

The rapid development of molecular biology has promoted the development of plant systematics. The main basis of plant systematics is the "tree of life" of Darwin's theory of evolution [1], based on the theory of "common ancestor" and "germogenesis" [8]. The system can show phylogenetic relationships among different beings, but cannot show evolutionary relationships, which can lead to a subjective system sometimes [1]. For example, the APG system considers the family Amborellaceae Pinch. as the most primitive angiosperm and establish taxonomic taxa such as Eudicots and Core eudicots [8]. These unscientific conclusions and taxa are concrete manifestations of the theoretical limitations. The phylogenetic theory itself offers no scientific ideas and methods for understanding the evolutionary veins of fruit plants, because how to find and process some "noise [5]" occurred in the evolution of fruit plants is still a puzzle (here, "noise" means DNA sequences not having important evolutionary significance).

How scientifically settle the puzzle of origin of fruit plants? The chloroplast genomic sequences of three primitive species of Yulania subsect. Cylindricae (Spongb.) D. L. Fu, subsect. comb. nov. (Magnoliaceae Juss.) were determined and compared with some primitive taxa by means of the typical algorithm based on the evolutionary continuity principle [1]. The results and analyses are as follows.

\section{Materials and Methods}

\subsection{Chloroplast Genome Sequencing, Assembly and Annotation}

Total genomic DNA was isolated from silica-dried leaves of three species of Yulania subsect. Cylindricae: Y. cylindrica (Wils.) D. L. Fu from Xiuning county of Anhui province, Y. shizhenii D. L. Fu et F. W. Li from Chengdu City of Sichuan province and new species of Y. puberula D. L. Fu from Mountain Wudang in Hubei Province, using a modified CTAB method [9]. After quantification and qualification, a paired-end library was constructed, and high-throughput sequencing was performed using the Illumina Hiseq 2500 platform (Lemont, IL, USA). After cleaning the raw data, all remaining high quality sequences were assembled into contigs using de novo assembly, and the complete chloroplast genomes were assembled using the software SPAdes v3.9.0. The circular map of fully annotated genomes were drawn in OGDRAWv1.2 [10]. All three chloroplast genomes of Yulania subsect. Cylindricae were deposited in the GenBank database.

\subsection{Evolutionary Analyses of Chloroplast Complete Genomes}

By analyzing the morphological characters using evolutionary continuity principle of Evolutionomy [1], the chloroplast genomes of three species of Yulania subsect. Cylindricae (Spongb.) D. L. Fu, subsect. comb. nov. (Magnoliaceae) were compared with other primitive groups such as Amborellaceae Pinch., Winteraceae Lindley, Trochodendraceae Prant., in order to scientifically determine the evolutionary position of Yulania subsect. Cylindricae (Spongb.) D. L. Fu in the evolution of fruit plants. The evolutionary analyses of chloroplast genomes mainly adopted the typical algorithm, by comparing EVS = the evolutionary similarities between the designated type and species or taxa, and $\mathrm{PHS}=$ the phylogenetic similarity between the designated type and species or taxa, to determine the relatively evolutionary relationship among different taxa. The formulas are as follows:

\section{$\mathrm{EVS}=\mathrm{SEVL} / \mathrm{AEVL}$}

EVS = evolutionary similarity; SEVL = same evolutionary loci between the type and taxon; $\mathrm{AEVL}=$ all evolutionary loci between the type and taxa (evolutionary loci statistics just including SNB of transitions and transversions in the middle of barcode; SNB = Single Nucleotide Barcode)

\section{$\mathrm{PHS}=\mathrm{SPHL} / \mathrm{APHL}$}

PHS = phylogenetic similarity; SPHL $=$ same phylogenetic loci between the type and taxon; APHL = all phylogenetic loci of the type (the loci statistics according NB; NB =Nucleotide Barcode)

\section{Chloroplast Genomes of Three Species of Yulania Subsect. Cylindricae}

The chloroplast genomes of three species of Yulania, similar to the chloroplast genomes of other higher plants, contains two inverted repeats, IRA and IRB, which make the entire genes component into four parts, the remaining LSC large single copy area and SSC small single copy area. For the basic information of genomic length, partition length, GC content, and number of coding genes of chloroplasts of three species, see Table 1 and Figure 1. 
Table 1. Basic characteristics of chloroplast genomes of three species of Yulania subsect. Cylindricae.

\begin{tabular}{llll}
\hline $\begin{array}{l}\text { Chloroplast } \\
\text { genome }\end{array}$ & $\begin{array}{l}\text { Yulania } \\
\text { cylindrica }\end{array}$ & $\begin{array}{l}\text { Yulania } \\
\text { puberula }\end{array}$ & $\begin{array}{l}\text { Yulania } \\
\text { shizhenii }\end{array}$ \\
\hline Total length & $160,079 \mathrm{bp}$ & $160,067 \mathrm{bp}$ & $160,062 \mathrm{bp}$ \\
LSC & $88,118 \mathrm{bp}$ & $88,108 \mathrm{bp}$ & $88,103 \mathrm{bp}$ \\
SSC & $18,779 \mathrm{bp}$ & $18,779 \mathrm{bp}$ & $18,779 \mathrm{bp}$ \\
IR & $26,591 \mathrm{bp}$ & $26,590 \mathrm{bp}$ & $26,590 \mathrm{bp}$ \\
GC $\%$ & $39.3 \%$ & $39.3 \%$ & $39.3 \%$ \\
\hline
\end{tabular}

Table 2. List of genes present in the chloroplast genomes of three species of Yulania subsect. Cylindricae.

\begin{tabular}{ll}
\hline Function & Group of genes Gene names \\
\hline ATP synthase & $a t p \mathrm{~A}, a t p \mathrm{~B}, a t p \mathrm{E}, a t p \mathrm{~F}^{*}, a t p \mathrm{H}, a t p \mathrm{I}$ \\
Cytochrome b/f complex & $p e t \mathrm{~A}, p e \mathrm{~B}, p e t \mathrm{D}, p e t \mathrm{G}, p e t \mathrm{~L}, p e t \mathrm{~N}$ \\
& $n d h \mathrm{~A}^{*}, n d h \mathrm{~B}^{*}, n d h \mathrm{C}, n d h \mathrm{D}, n d h \mathrm{E}, n d h \mathrm{~F}$, \\
NADH dehydrogenase & $n d h \mathrm{G}, n d h \mathrm{H}, n d h \mathrm{I}, n d h \mathrm{~J}, n d h \mathrm{~K}$ \\
Photosystem I & $p s a \mathrm{~A}, p s a \mathrm{~B}, p s a \mathrm{C}, p s a \mathrm{I}, p s a \mathrm{~J}$ \\
Photosystem II & $p s b \mathrm{~A}, p s b \mathrm{~B}, p s b \mathrm{C}, p s b \mathrm{D}, p s b \mathrm{E}, p s b \mathrm{~F}, p s b \mathrm{H}$, \\
Proteins of unknown & $p s b \mathrm{I}, p s b \mathrm{~J}, p s b \mathrm{~K}, p s b \mathrm{~L}, p s b \mathrm{M}, p s b \mathrm{~N}, p s b \mathrm{~T}, p s b \mathrm{Z}$ \\
function & $y c f 1^{1}, y c f 2, y c f 3^{* *}, y c f 4$ \\
Ribosomal proteins (SSU) & $r p s 2, r p s 3, r p s 4, r p s 7, r p s 8, r p s 11, r p s 12^{\#}$, \\
& $r p s 14, r p s 15, r p s 16, r p s 18, r p s 19$ \\
Ribosomal proteins (LSU) & $r p l 2^{*}, r p l 14, r p l 16, r p l 20, r p l 22, r p l 23, r p l 32$, \\
Ribosomal RNAs & $r p l 33, r p l 36$ \\
RNA polymerase & $r r n 4.5^{1}, r r n 5^{1}, r r n 16^{1}, r r n 23^{1}$ \\
& $r p o \mathrm{~A}, r p o \mathrm{~B}, r p o \mathrm{C} 1^{*}, r p o \mathrm{C} 2$ \\
\hline
\end{tabular}

\begin{tabular}{|c|c|}
\hline Function & Group of genes Gene names \\
\hline \multirow[t]{5}{*}{ Other genes } & $a c c \mathrm{D}, c c s \mathrm{~A}, c e m \mathrm{~A}, c l p \mathrm{P}^{* *}, m a t \mathrm{~K}, r b c \mathrm{~L}, \inf \mathrm{A}$ \\
\hline & tRNA-Lys*, tRNA-Gln, tRNA-Ser, \\
\hline & tRNA-Gly*, tRNA-Arg, tRNA-Cys, \\
\hline & tRNA-Asp, tRNA-Tyr, tRNA-Glu, tRNA-Thr, \\
\hline & $\begin{array}{l}\text { tRNA-Ser, tRNA-Gly, tRNA-Met, tRNA-Ser, } \\
\text { tRNA-Thr, tRNA-Leu, tRNA-Phe, tRNA-Val, }\end{array}$ \\
\hline \multirow[t]{5}{*}{ Transfer RNAs } & tRNA-Met, tRNA-Trp, tRNA-Pro, tRNA-Ile, \\
\hline & tRNA-Leu*, tRNA-Val*, tRNA-His, \\
\hline & tRNA-Ile*1, tRNA-Ala*1, tRNA-Arg ${ }^{1}$, \\
\hline & tRNA-Asn ${ }^{1}$, tRNA-Leu, tRNA-Asn, \\
\hline & tRNA-Arg, t RNA-Ala, tRNA-Ile, tRNA-His \\
\hline Other & ORF302 ${ }^{1}$ \\
\hline
\end{tabular}

*gene containing a single intron, **gene containing two introns, \#trans-splicing gene, ${ }^{1}$ Two gene copies in Irs

The complete chloroplast genomes of Yulania cylindrica, Y. puberula and Y. shizhenii are 160,079bp, 160,067bp and 160,062 bp in length, respectively. They are all encoded128 genes. Among them, there are 83 protein coding genes, 8 rRNA genes and 37 tRNAs. trnK-UUU, rps 16 , trnG-UCC, atp $\mathrm{F}, r p o \mathrm{C} 1$, trnL-UAA, trnV-UAC, pet $\mathrm{B}$, pet $\mathrm{D}, r p l 16, r p l 2$, $n d h \mathrm{~B}, \operatorname{trnI}-\mathrm{GAU}, \operatorname{trn} \mathrm{A}-\mathrm{UGC}, n d h \mathrm{~A}$ genes with an intron for each gene, $c l p \mathrm{P}, y c f 3$ gene with two introns and the $r p s 12$ is trans-splicing gene, see Table 2 and Figure 1.

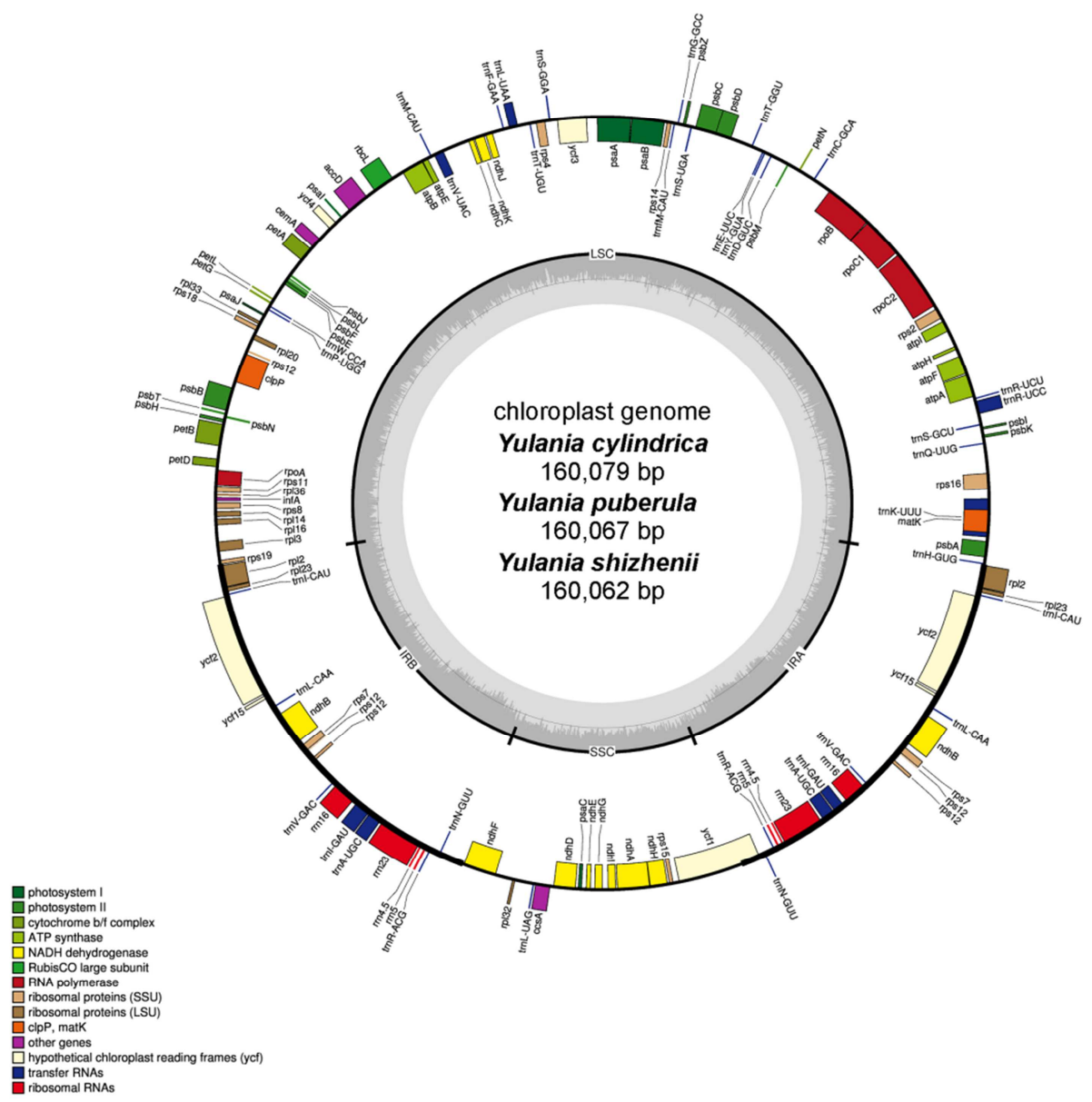

Figure 1. Chloroplast genome map of 3 species of Yulania subsect. Cylindricae (Magnoliaceae). The genes inside and outside the circle are transcribed in the clockwise and counterclockwise directions, respectively. Genes belonging to different functional groups are shown in different colors. The thick lines indicate the extent of the inverted repeats (IRA and IRB) that separate the genomes into small single-copy (SSC) and large single-copy (LSC) regions. 


\section{Origin of Fruit Plants}

According to the evolutionary continuity principle, it is known that Fructophyta D. L. Fu \& H. Fu originated from Gymnospermophyta D. L. Fu \& H. Fu [1], but we don't know which taxa of Gymnospermophyta have the closest evolutionary relationships with fruit plants especially about the main evolutionary vein of origin and evolution of fruits. Because the new species Yulania puberula D. L. Fu of subsect. Cylindricae (Spongb.) D. L. Fu has the most primitive peruloid bracts (see Figure 3(7)), so it is selected as the type of fruit plants and calculated the evolutionary similarity and the phylogenetic similarity among the samples of Gymnospermophyta from NCBI, the results are shown in Table 3.
It can be seen from Table 3 that there are 1,69 1evolutionary loci $(\mathrm{SNB}=21 \mathrm{bp}$ ) between the species of Yulania puberula and gymnosperms, of which 453 are the same with the species of Ginkgo biloba and the EVS is 0.2679 , which is higher than other gymnosperm samples. Therefore Yulania puberula and Ginkgo biloba has the closest relatively evolutionary relationship. PHS between Yulania puberula and Ginkgo biloba ( $\mathrm{PHS}=0.0548$ ) is also obviously higher than other samples of gymnosperms. According to the evolutionary continuity principle, it can be determined that fruit plants originated from Ginkgoopsida, not from Cycadopsida thought by the euanthium-theory or Chlamydopsermopsida by the pseudoanthium- theory.

Table 3. EVS and PHS of chloroplast genomes between Yulania puberula and different species of Gymnospermophyta from NCBI.

\begin{tabular}{|c|c|c|c|c|c|c|c|c|c|c|}
\hline Phylum & Class & $\begin{array}{l}\text { Species name and Genomic } \\
\text { number of NCBI }\end{array}$ & $\begin{array}{l}\text { EVL } \\
\text { /19bp }\end{array}$ & EVS & $\begin{array}{l}\text { PHL } \\
/ 19 b p\end{array}$ & PHS & $\begin{array}{l}\text { EVL } \\
/ 21 \text { bp }\end{array}$ & EVS & $\begin{array}{l}\text { PHL } \\
\text { /21bp }\end{array}$ & PHS \\
\hline Fructophyta & Magnoliopsida & Yulania puberula & 2022 & 1 & 133320 & 1 & 1691 & 1 & 133377 & 1 \\
\hline Gymnospermophyta & Ginkgoopsida & Ginkgo biloba_JN867578.1 & 542 & 0.2681 & 8456 & 0.0634 & 453 & 0.2679 & 7310 & 0.0548 \\
\hline Gymnospermophyta & Cycadopsida & $\begin{array}{l}\text { Cycas } \\
\text { panzhihuaensis_NC031413.1 }\end{array}$ & 528 & 0.2611 & 8506 & 0.0638 & 451 & 0.2667 & 7280 & 0.0546 \\
\hline Gymnospermophyta & Cycadopsida & Cycas revoluta_NC020319.1 & 524 & 0.2591 & 8484 & 0.0636 & 446 & 0.2637 & 7264 & 0.0545 \\
\hline Gymnospermophyta & Cycadopsida & Cycas taitungensis_AP009339.1 & 521 & 0.2577 & 8457 & 0.0634 & 443 & 0.262 & 7250 & 0.0544 \\
\hline Gymnospermophyta & Coniferopsida & Pinus tabuliformis_NC028531.1 & 410 & 0.2028 & 6409 & 0.0481 & 356 & 0.2105 & 5565 & 0.0417 \\
\hline Gymnospermophyta & Coniferopsida & $\begin{array}{l}\text { Cunninghamia } \\
\text { lanceolata_NC021437.1 }\end{array}$ & 326 & 0.1612 & 5078 & 0.0381 & 269 & 0.1591 & 4214 & 0.0316 \\
\hline Gymnospermophyta & Taxopsida & $\begin{array}{l}\text { Cephalotaxus } \\
\text { oliveri_NC021110.1 }\end{array}$ & 289 & 0.1429 & 4586 & 0.0344 & 242 & 0.1431 & 3723 & 0.0279 \\
\hline Gymnospermophyta & Taxopsida & Taxus mairei_NC020321.1 & 263 & 0.1301 & 4433 & 0.0333 & 217 & 0.1283 & 3628 & 0.0272 \\
\hline Gymnospermophyta & Coniferopsida & Cupressus gigantea_NC028155.1 & 251 & 0.1241 & 4443 & 0.0333 & 206 & 0.1218 & 3661 & 0.0274 \\
\hline Gymnospermophyta & Chlamydopsermopsida & $\begin{array}{l}\text { Welwitschia } \\
\text { mirabilis_NC010654.1 }\end{array}$ & 230 & 0.1137 & 3819 & 0.0286 & 184 & 0.1088 & 3245 & 0.0243 \\
\hline Gymnospermophyta & Chlamydopsermopsida & Gnetum montanum_NC021438.1 & 173 & 0.0856 & 3361 & 0.0252 & 140 & 0.0828 & 2870 & 0.0215 \\
\hline Gymnospermophyta & Chlamydopsermopsida & Gnetum ula_AP014923.1 & 173 & 0.0856 & 3363 & 0.0252 & 141 & 0.0834 & 2875 & 0.0216 \\
\hline Gymnospermophyta & Chlamydopsermopsida & Ephedra foeminea_NC029347.1 & 163 & 0.0806 & 3220 & 0.0242 & 132 & 0.0781 & 2716 & 0.0204 \\
\hline
\end{tabular}

\section{Relatively Original Species of Fruit Plants}

\subsection{Relatively Evolutionary Relationships Among Different Classes or Subclasses of Fructophyta}

For only one species survived in Ginkgoopsida, so Ginkgo biloba is consequentially selected as the type of Gymnospermophyta. To represent 5 classes and 20 subclasses, 22 species were selected and calculated the evolutionary similarity and the phylogenetic similarity with the type, the results are shown in Table 4.

It can be seen from Table 4 that EVS and PHS between Ginkgo biloba and Yulania puberula is 0.2776 and 0.0608 respectively, obvious higher than other species such as Trochodendron aralioides and Amborella trichopoda. So Magnoliidae is primitive subclass of fruit plants. it is not supported that Trochodendron Siebold \& Zucc. are original family in H. D. Zhang system and Amborella Baill. Are original family in APG system by chloroplast genomic evolutionomy.

\subsection{Relatively Evolutionary Relationships Among Different Families of Magnoliidae}

For only 10 families having chloroplast complete genomes in NCBI, 10 species were selected and calculated the EVS and PHS with Ginkgo biloba, the results are shown in Table 5.

It can be seen from Table5 that EVS and PHS between Ginkgo biloba and Yulania puberula is 0.2475 and 0.0608 respectively, obvious higher than other species such as Drimys granadensis. So Magnoliaceae is the most primitive family of fruit plants. It is not supported that Winteraceae R. Br. ex Lindl. were the most primitive family in Takhtajan system and Cronquist system by chloroplast genomic evolutionomy. 
Table 4. EVS and PHS of chloroplast genomes between Ginkgo biloba and different species of Fructophyta from NCBI.

\begin{tabular}{|c|c|c|c|c|c|c|}
\hline Phylum & Class and Subclass & Species name and Genomic number of NCBI & $\begin{array}{l}\text { EVL } \\
/ 19 b p \\
\end{array}$ & EVS & $\begin{array}{l}\text { PHL } \\
/ 19 b p \\
\end{array}$ & PHS \\
\hline Gymnospermophyta & Ginkgoopsida & Ginkgo biloba_JN867578.1 & 2554 & 1 & 139127 & 1 \\
\hline Fructophyta & Magnoliopsida Magnoliidae & Yulania puberula & 709 & 0.2776 & 8457 & 0.0608 \\
\hline Fructophyta & Magnoliopsida Trochodendridae & Trochodendron aralioides_KC608753.1 & 659 & 0.258 & 8004 & 0.0575 \\
\hline Fructophyta & Rasopsida Hamamelididae & Hamamelis mollis_NC037881.1 & 653 & 0.2557 & 7760 & 0.0558 \\
\hline Fructophyta & Magnoliopsida Piperidae & Saruma henryi_NC039933.1 & 636 & 0.249 & 7953 & 0.0572 \\
\hline Fructophyta & Monocotyledonopsida Arecidae & Phytelephas aequatorialis_NC029957.1 & 620 & 0.2428 & 7927 & 0.057 \\
\hline Fructophyta & Magnoliopsida Ranunculidae & Nandina domestica_NC008336.1 & 597 & 0.2338 & 7820 & 0.0562 \\
\hline Fructophyta & Magnoliopsida Nymphaeidae & Amborella trichopoda_NC005086.1 & 589 & 0.2306 & 8442 & 0.0607 \\
\hline Fructophyta & Monocotyledonopsida Bromeliidae & Ananas comosus_NC026220.1 & 570 & 0.2232 & 7550 & 0.0543 \\
\hline Fructophyta & Monocotyledonopsida Commelinidae & Heliconia collinsiana_NC020362.1 & 568 & 0.2224 & 7685 & 0.0552 \\
\hline Fructophyta & Rasopsida Cornidae & Swida controversa_KU852492.1 & 565 & 0.2212 & 7554 & 0.0543 \\
\hline Fructophyta & Rasopsida Asteridae & Menyanthes trifoliata_NC041436.1 & 555 & 0.2173 & 7295 & 0.0524 \\
\hline Fructophyta & Rasopsida Lamiidae & Ole exasperata_NC036985.1 & 538 & 0.2106 & 7212 & 0.0518 \\
\hline Fructophyta & Rasopsida Rosidae & Malus prunifolia_NC031163.1 & 525 & 0.2056 & 7028 & 0.0505 \\
\hline Fructophyta & Rasopsida Caryophyllidae & Nepenthes mirabilis_MH346374.1 & 521 & 0.204 & 7170 & 0.0515 \\
\hline Fructophyta & Leguminopsida & Ceratonia siliqua_KJ468096.1 & 512 & 0.2005 & 7052 & 0.0507 \\
\hline Fructophyta & Monocotyledonopsida Acoridae & Acorus calamus_NC007407.1 & 510 & 0.1997 & 7306 & 0.0525 \\
\hline Fructophyta & Rasopsida Euphorbiidae & Hevea brasiliensis_NC015308.1 & 508 & 0.1989 & 7022 & 0.0505 \\
\hline Fructophyta & Monocotyledonopsida Alismatidae & Zantedeschia aethiopica_NC035499.1 & 507 & 0.1985 & 7184 & 0.0516 \\
\hline Fructophyta & Monocotyledonopsida Orchididae & Elleanthus sodiroi_KR260986.1 & 494 & 0.1934 & 7166 & 0.0515 \\
\hline Fructophyta & Rasopsida Malviidae & Tilia paucicostata_KT894775.1 & 486 & 0.1903 & 6894 & 0.0496 \\
\hline Fructophyta & Rasopsida Geraniidae & Hypseocharis bilobata_NC023260.1 & 365 & 0.1429 & 5802 & 0.0417 \\
\hline Fructophyta & Scutellopsida & Bambusa multiplex_NC024668.1 & 318 & 0.1245 & 5355 & 0.0385 \\
\hline
\end{tabular}

Table 5. EVS and PHS of chloroplast genomes between Ginkgo biloba and different families of Magnoliidae.

\begin{tabular}{|c|c|c|c|c|c|c|c|}
\hline Phylum & $\begin{array}{l}\text { Class or } \\
\text { subclass }\end{array}$ & Family & $\begin{array}{l}\text { Species name and Genomic number of } \\
\text { NCBI }\end{array}$ & $\begin{array}{l}\text { EVL } \\
/ 19 b p\end{array}$ & EVS & $\begin{array}{l}\text { PHL } \\
/ 19 b p\end{array}$ & PHS \\
\hline Gymnospermophyta & Ginkgoopsida & Ginkgoaceae & Ginkgo biloba_JN867578.1 & 1471 & 1 & 139127 & 1 \\
\hline Fructophyta & Magnoliidae & Magnoliaceae & Yulania puberula & 364 & 0.2475 & 8457 & 0.0608 \\
\hline Fructophyta & Magnoliidae & Winteraceae & Drimys granadensis_DQ887676.1 & 325 & 0.2209 & 8093 & 0.0582 \\
\hline Fructophyta & Magnoliidae & Calycanthaceae & Calycanthus chinensis_NC037504.1 & 319 & 0.2169 & 7963 & 0.0572 \\
\hline Fructophyta & Magnoliidae & Chloranthaceae & Chloranthus japonicus_NC026565.1 & 318 & 0.2162 & 8185 & 0.0588 \\
\hline Fructophyta & Magnoliidae & Myristicaceae & Horsfieldia pandurifolia_MH445411.1 & 311 & 0.2114 & 8005 & 0.0575 \\
\hline Fructophyta & Magnoliidae & Cryptocaryaceae & Cryptocarya chinensis_NC036002.1 & 301 & 0.2046 & 7651 & 0.055 \\
\hline Fructophyta & Magnoliidae & Lauraceae & Cinnamomum camphora_NC035882.1 & 286 & 0.1944 & 7663 & 0.0551 \\
\hline Fructophyta & Magnoliidae & Hernandiaceae & Hernandia nymphaeifolia_MG838431.1 & 277 & 0.1883 & 7407 & 0.0532 \\
\hline Fructophyta & Magnoliidae & Neocinnamomaceae & Neocinnamomum mekongense_MF686120.1 & 263 & 0.1788 & 7362 & 0.0529 \\
\hline Fructophyta & Magnoliidae & Annonaceae & Annona cherimola_NC030166.1 & 224 & 0.1523 & 6570 & 0.0472 \\
\hline
\end{tabular}

\subsection{Relatively Evolutionary Relationships Among Different Genera of Magnoliaceae}

To scientifically reflect the relatively evolutionary relationships among different genera of Magnoliaceae, 28 species having chloroplast complete genomes in NCBI were selected and calculated the evolutionary similarity and the phylogenetic similarity with the type of Ginkgo biloba, the results are shown in Table 6.

It can be seen from Table 6 that EVS between Ginkgo biloba and Yulania diva? is 0.0859 obvious higher than other genera and other species such as Magnolia grandiflora, Liriodendron tulipifera and Michelia laevifolia. So it can be concluded that Yulania Spach is the most primitive genus of Magnoliaceae. It is not supported that Magnolia L. is the most primitive genus in Hutchinson system and $\mathrm{C}$. $\mathrm{Y}$. $\mathrm{Wu}$ system (Manglietia Blum.=Magnolia L.).

\subsection{Relatively Evolutionary Relationships Among Species of Genus Yulania}

In order to determine the relatively evolutionary relationship among the species of genus of Yulania, the EVS and PHS between Ginkgo biloba and 10 species of Yulania Spach were calculated, and the results are shown in Table 7:

It can be seen from Table 7 that Yulania puberula and Yulania cylindrica have the closest relatively evolutionary relationships with Ginkgo biloba, and the evolutionary similarities are same as $\mathrm{EVS}=0.0255$, but PHS between Yulania puberula and Ginkgo biloba is $\mathrm{PHS}=0.0713$, which is higher than $\mathrm{PHS}=0.0712$ between Yulania cylindrica and Ginkgo biloba, so Yulania puberula has the closest evolutionary relationship and phylogenetic relationship with Gingko biloba among species of the genus of Yulania Spach.

So it can be concluded that Magnoliaceae Juss. are the most primitive family, Yulania Spach is the most primitive 
genus and Yulania puberula is the most primitive species of Fructophyta.

Table 6. EVS and PHS of chloroplast genomes between Ginkgo biloba and different species of Magnoliaceae Juss.

\begin{tabular}{|c|c|c|c|c|c|}
\hline Phylum & Species name and Genomic number of NCBI & EVL/17bp & EVS & PHL/17bp & PHS \\
\hline Gymnospermophyta & Ginkgo biloba_JN867578.1 & 1153 & 1 & 138938 & 1 \\
\hline Fructophyta & Yulania diva? ${ }^{-} \mathrm{NC} 023242.1$ & 99 & 0.0859 & 9900 & 0.0713 \\
\hline Fructophyta & Yulania puberūa & 97 & 0.0841 & 9905 & 0.0713 \\
\hline Fructophyta & Yulania cylindrica & 97 & 0.0841 & 9894 & 0.0712 \\
\hline Fructophyta & Yulania salicifolia NC023240.1 & 97 & 0.0841 & 9909 & 0.0713 \\
\hline Fructophyta & Magnolia grandiflora_NC020318.1 & 97 & 0.0841 & 9941 & 0.0715 \\
\hline Fructophyta & Magnolia dealbata_NC023235.1 & 96 & 0.0833 & 9883 & 0.0711 \\
\hline Fructophyta & Yulania shizhenii & 96 & 0.0833 & 9903 & 0.0713 \\
\hline Fructophyta & Liriodendron tulipifera_DQ899947.1 & 96 & 0.0833 & 9901 & 0.0713 \\
\hline Fructophyta & Magnolia officinalis_NC020316.1 & 95 & 0.0824 & 9906 & 0.0713 \\
\hline Fructophyta & Yulania denudata_JN867577.1 & 95 & 0.0824 & 9894 & 0.0712 \\
\hline Fructophyta & Yulania liliiflora_JX280397.1 & 95 & 0.0824 & 9658 & 0.0695 \\
\hline Fructophyta & Yulania biondii_KY085894.1 & 95 & 0.0824 & 9886 & 0.0712 \\
\hline Fructophyta & Magnolia kwangsiensis_HM775382.1 & 95 & 0.0824 & 9922 & 0.0714 \\
\hline Fructophyta & Michelia laevifolia_NC035956.1 & 95 & 0.0824 & 9909 & 0.0713 \\
\hline Fructophyta & Liriodendron chinense_NC030504.1 & 95 & 0.0824 & 9903 & 0.0713 \\
\hline Fructophyta & Yulania acuminata_JX280391.1 & 94 & 0.0815 & 9905 & 0.0713 \\
\hline Fructophyta & Magnolia pyramidata_NC023236.1 & 93 & 0.0807 & 9920 & 0.0714 \\
\hline Fructophyta & Magnolia sinica_NC023241.1 & 93 & 0.0807 & 9887 & 0.0712 \\
\hline Fructophyta & Magnolia dandyi_NC_037004.1 & 92 & 0.0798 & 9869 & 0.071 \\
\hline Fructophyta & Magnolia tripetala_NCD24027.1 & 92 & 0.0798 & 9879 & 0.0711 \\
\hline Fructophyta & Yulania kobus_NC 023237.1 & 92 & 0.0798 & 9877 & 0.0711 \\
\hline Fructophyta & Michelia odora_NC023239.1 & 92 & 0.0798 & 9868 & 0.071 \\
\hline Fructophyta & Magnolia yunnanensis_NC024545.1 & 92 & 0.0798 & 9896 & 0.0712 \\
\hline Fructophyta & Magnolia glaucifolia_Ne $\mathrm{N} 037003.1$ & 91 & 0.0789 & 9876 & 0.0711 \\
\hline Fructophyta & Magnolia duclouxii_NC037002.1 & 91 & 0.0789 & 9899 & 0.0712 \\
\hline Fructophyta & Magnolia fordiana_MF-M990562.1 & 91 & 0.0789 & 9871 & 0.071 \\
\hline Fructophyta & Michelia cathcartii NC023234.1 & 90 & 0.0781 & 9876 & 0.0711 \\
\hline Fructophyta & Magnolia insignis MF990566.1 & 89 & 0.0772 & 9893 & 0.0712 \\
\hline
\end{tabular}

Table 7. EVS and PHS between Ginkgo biloba and different species of Yulania Spach.

\begin{tabular}{|c|c|c|c|c|}
\hline Species name and Genomic number of NCBI & EVL/17bp & EVS & PHL/17bp & PHS \\
\hline Ginkgo biloba_JN867578.1 & 982 & 1 & 138938 & 1 \\
\hline Yulania puberula & 25 & 0.0255 & 9905 & 0.0713 \\
\hline Yulania cylindrica & 25 & 0.0255 & 9894 & 0.0712 \\
\hline Yulania shizhenii & 24 & 0.0244 & 9903 & 0.0713 \\
\hline Yulania diva?_NC023242.1 & 24 & 0.0244 & 9900 & 0.0713 \\
\hline Yulania salicifolia_NC023240.1 & 24 & 0.0244 & 9909 & 0.0713 \\
\hline Yulania liliiflora_JX280397.1 & 23 & 0.0234 & 9658 & 0.0695 \\
\hline Yulania biondii_KY085894.1 & 23 & 0.0234 & 9886 & 0.0712 \\
\hline Yulania acuminata_JX280391.1 & 22 & 0.0224 & 9905 & 0.0713 \\
\hline Yulania denudata_JN867577.1 & 22 & 0.0224 & 9894 & 0.0712 \\
\hline Yulania kobus_NC 023237.1 & 21 & 0.0214 & 9877 & 0.0711 \\
\hline
\end{tabular}

Table 8. PHS and EVS of chloroplast genomes between Yulania denudata and different species of Magnoliaceae Juss.

\begin{tabular}{|c|c|c|c|c|}
\hline Species name and cpDNA number of NCBI & PHL/17bp & PHS & EVL/17bp & EVS \\
\hline Yulania denudata_JN867577.1 & 133206 & 1 & 4155 & 1 \\
\hline Yulania cylindrica & 131736 & 0.99 & 4084 & 0.98 \\
\hline Yulania puberula & 131551 & 0.99 & 4074 & 0.98 \\
\hline Yulania shizhenii & 131460 & 0.99 & 4068 & 0.98 \\
\hline Yulania liliiflora_JX280397.1 & 129270 & 0.97 & 3979 & 0.96 \\
\hline Yulania salicifolia_NC023240.1 & 127070 & 0.95 & 3756 & 0.90 \\
\hline Yulania diva? NC023242.1 & 126820 & 0.95 & 3722 & 0.90 \\
\hline Yulania biondii KY085894.1 & 126313 & 0.95 & 3667 & 0.88 \\
\hline Yulania kobus $\mathrm{NC} 023237.1$ & 125689 & 0.94 & 3632 & 0.87 \\
\hline Yulania acuminata_JX280391.1 & 123659 & 0.93 & 3505 & 0.84 \\
\hline Magnolia yunnanensis_NC024545.1 & 122609 & 0.92 & 3415 & 0.82 \\
\hline Magnolia sinica_NC023241.1 & 122465 & 0.92 & 3389 & 0.82 \\
\hline Magnolia dealbata_NC023235.1 & 121824 & 0.91 & 3366 & 0.81 \\
\hline Magnolia fordiana_MF990562.1 & 121647 & 0.91 & 3332 & 0.80 \\
\hline Magnolia dandyi_NC037004.1 & 121609 & 0.91 & 3325 & 0.80 \\
\hline Magnolia insignis_MF990566.1 & 121607 & 0.91 & 3329 & 0.80 \\
\hline
\end{tabular}




\begin{tabular}{|c|c|c|c|c|}
\hline Species name and cpDNA number of NCBI & PHL/17bp & PHS & EVL/17bp & EVS \\
\hline Magnolia duclouxii_NC037002.1 & 121580 & 0.91 & 3332 & 0.80 \\
\hline Magnolia tripetala_NC024027.1 & 121578 & 0.91 & 3316 & 0.80 \\
\hline Michelia laevifolia_NC035956.1 & 121575 & 0.91 & 3328 & 0.80 \\
\hline Magnolia kwangsiensis_HM775382.1 & 121536 & 0.91 & 3361 & 0.81 \\
\hline Magnolia glaucifolia_NC037003.1 & 121499 & 0.91 & 3324 & 0.80 \\
\hline Magnolia grandiflora_NC020318.1 & 121332 & 0.91 & 3326 & 0.80 \\
\hline Magnolia pyramidata $\mathrm{NC} 023236.1$ & 121260 & 0.91 & 3321 & 0.80 \\
\hline Michelia cathcartii NC023234.1 & 121126 & 0.91 & 3307 & 0.80 \\
\hline Magnolia officinalis_NC020316.1 & 121121 & 0.91 & 3273 & 0.79 \\
\hline Michelia odora_NC023239.1 & 121014 & 0.91 & 3294 & 0.79 \\
\hline Liriodendron tulipifera_DQ899947.1 & 103965 & 0.78 & 2039 & 0.49 \\
\hline Liriodendron chinense_NC030504.1 & 103689 & 0.78 & 2009 & 0.48 \\
\hline
\end{tabular}

Table 9. PHS and EVS of chloroplast genomes between Magnolia fordiana and different species of Magnoliaceae Juss.

\begin{tabular}{|c|c|c|c|c|}
\hline Species name and cpDNA number of NCBI & PHL/17bp & PHS & EVL17bp & EVS \\
\hline Magnolia fordiana_MF990562.1 & 133163 & 1 & 4187 & 1 \\
\hline Magnolia dandyi_NC037004.1 & 132460 & 0.99 & 4136 & 0.99 \\
\hline Magnolia glaucifolia_NC037003.1 & 132385 & 0.99 & 4134 & 0.99 \\
\hline Magnolia duclouxii_NC037002.1 & 129855 & 0.98 & 3955 & 0.94 \\
\hline Magnolia insignis_MF990566.1 & 129850 & 0.98 & 3942 & 0.94 \\
\hline Magnolia tripetala_NC024027.1 & 125593 & 0.94 & 3611 & 0.86 \\
\hline Magnolia officinalis_NC020316.1 & 124347 & 0.93 & 3534 & 0.84 \\
\hline Magnolia yunnanensis_NC024545.1 & 123790 & 0.93 & 3481 & 0.83 \\
\hline Magnolia sinica_NC023241.1 & 123496 & 0.93 & 3445 & 0.82 \\
\hline Magnolia kwangsiensis_HM775382.1 & 123473 & 0.93 & 3479 & 0.83 \\
\hline Magnolia grandiflora_NC020318.1 & 123454 & 0.93 & 3500 & 0.84 \\
\hline Magnolia pyramidata_NC023236.1 & 123322 & 0.93 & 3462 & 0.83 \\
\hline Yulania acuminata_JX280391.1 & 122861 & 0.92 & 3429 & 0.82 \\
\hline Michelia laevifolia_NC035956.1 & 122773 & 0.92 & 3397 & 0.81 \\
\hline Michelia odora_NC023239.1 & 122171 & 0.92 & 3349 & 0.80 \\
\hline Yulania diva?_NC023242.1 & 122135 & 0.92 & 3350 & 0.80 \\
\hline Yulania puberula & 122115 & 0.92 & 3356 & 0.80 \\
\hline Yulania cylindrica & 122078 & 0.92 & 3344 & 0.80 \\
\hline Yulania biondii_KY085894.1 & 122009 & 0.92 & 3339 & 0.80 \\
\hline Michelia cathcartii_NC023234.1 & 121998 & 0.92 & 3322 & 0.79 \\
\hline Yulania shizhenii & 121947 & 0.92 & 3344 & 0.80 \\
\hline Yulania denudata_JN867577.1 & 121646 & 0.91 & 3331 & 0.80 \\
\hline Yulania kobus_NC023237.1 & 121557 & 0.91 & 3312 & 0.79 \\
\hline Yulania liliiflora_JX280397.1 & 121203 & 0.91 & 3359 & 0.80 \\
\hline Liriodendron tulipifera_DQ899947.1 & 105598 & 0.79 & 2126 & 0.51 \\
\hline Liriodendron chinense_NC030504.1 & 105223 & 0.79 & 2087 & 0.50 \\
\hline
\end{tabular}

Table 10. PHS and EVS of chloroplast genomes between Michelia odora and different species of Magnoliaceae Juss.

\begin{tabular}{|c|c|c|c|c|}
\hline Species name and cpDNA number of NCBI & PHL/17bp & PHS & EVL/17bp & EVS \\
\hline Michelia odora_NC023239.1 & 133199 & 1 & 4146 & 1 \\
\hline Michelia laevifolia_NC035956.1 & 129813 & 0.97 & 3902 & 0.94 \\
\hline Michelia cathcartii_NC023234.1 & 125770 & 0.94 & 3607 & 0.87 \\
\hline Magnolia yunnanensis_NC024545.1 & 123016 & 0.92 & 3411 & 0.82 \\
\hline Magnolia sinica_NC023241.1 & 122776 & 0.92 & 3381 & 0.82 \\
\hline Magnolia dealbata_NC023235.1 & 122288 & 0.92 & 3370 & 0.81 \\
\hline Yulania acuminata_JX280391.1 & 122268 & 0.92 & 3373 & 0.81 \\
\hline Magnolia dandyi_NC037004.1 & 122155 & 0.92 & 3345 & 0.81 \\
\hline Magnolia tripetala_NC024027.1 & 122090 & 0.92 & 3341 & 0.81 \\
\hline Magnolia duclouxiii_NC037002.1 & 122078 & 0.92 & 3351 & 0.81 \\
\hline Yulania salicifolia_Ne-NC23240.1 & 122063 & 0.92 & 3362 & 0.81 \\
\hline Magnolia glaucifolia_NC037003.1 & 122048 & 0.92 & 3338 & 0.81 \\
\hline Magnolia insignis_MF-M90566.1 & 122030 & 0.92 & 3343 & 0.81 \\
\hline Magnolia kwangsiensis_HM775382.1 & 122021 & 0.92 & 3363 & 0.81 \\
\hline Yulania puberula & 121405 & 0.91 & 3308 & 0.80 \\
\hline Yulania cylindrica & 121381 & 0.91 & 3303 & 0.80 \\
\hline
\end{tabular}




\begin{tabular}{llll}
\hline Species name and cpDNA number of NCBI & PHL/17bp & PHS & EVL/17bp \\
\hline Yulania biondii_KY085894.1 & 121340 & 0.91 & 3280 \\
Yulania shizhenii & 121311 & 0.91 & 3304 \\
Magnolia grandiflora_NC020318.1 & 121033 & 0.91 & 3297 \\
Yulania denudata_JN867577.1 & 121014 & 0.91 & 3294 \\
Yulania kobus_NC023237.1 & 120843 & 0.91 & 3245 \\
Magnolia officinalis_NC020316.1 & 120742 & 0.91 & 3258 \\
Yulania liliiflora_JX280397.1 & 120661 & 0.91 & 3334 \\
Liriodendron tulipifera_DQ899947.1 & 104128 & 0.70 & 0.79 \\
Liriodendron chinense_NC030504.1 & 104000 & 0.78 & 0.79 \\
\hline
\end{tabular}

Table 11. PHS and EVS of cpGenomes between Liriodendron tulipifera and different species of Magnoliaceae Juss.

\begin{tabular}{|c|c|c|c|c|}
\hline Species name and cpDNA number of NCBI & PHL/17bp & PHS & EVL/17bp & EVS \\
\hline Liriodendron tulipifera_DQ899947.1 & 133246 & 1 & 3618 & 1 \\
\hline Liriodendron chinense_NC030504.1 & 123803 & 0.93 & 2995 & 0.83 \\
\hline Magnolia tripetala_NC024027.1 & 105693 & 0.79 & 2153 & 0.60 \\
\hline Magnolia insignis_MF990566.1 & 105662 & 0.79 & 2128 & 0.59 \\
\hline Magnolia duclouxii_NC037002.1 & 105656 & 0.79 & 2133 & 0.59 \\
\hline Magnolia dealbata_NC023235.1 & 105651 & 0.79 & 2131 & 0.59 \\
\hline Magnolia kwangsiensis_HM775382.1 & 105629 & 0.79 & 2172 & 0.60 \\
\hline Magnolia dandyi_NCON037004.1 & 105550 & 0.79 & 2125 & 0.59 \\
\hline Magnolia glaucifolia_NC037003.1 & 105471 & 0.79 & 2116 & 0.58 \\
\hline Magnolia yunnanensis_NC024545.1 & 105464 & 0.79 & 2128 & 0.59 \\
\hline Magnolia sinica_NC023241.1 & 105336 & 0.79 & 2117 & 0.59 \\
\hline Magnolia pyramidata_NC023236.1 & 105308 & 0.79 & 2134 & 0.59 \\
\hline Magnolia officinalis_NC020316.1 & 105033 & 0.79 & 2110 & 0.58 \\
\hline Yulania acuminata_JX280391.1 & 104930 & 0.79 & 2089 & 0.58 \\
\hline Magnolia grandiflora_NC020318.1 & 104838 & 0.79 & 2116 & 0.58 \\
\hline Yulania salicifolia_NC023240.1 & 104741 & 0.79 & 2100 & 0.58 \\
\hline Yulania diva?_NC023242.1 & 104483 & 0.78 & 2075 & 0.57 \\
\hline Michelia cathcartii NC023234.1 & 104322 & 0.78 & 2043 & 0.56 \\
\hline Yulania biondii_KȲ̄085894.1 & 104249 & 0.78 & 2061 & 0.57 \\
\hline Yulania cylindrica & 104133 & 0.78 & 2039 & 0.56 \\
\hline Michelia odora_NC023239.1 & 104130 & 0.78 & 2036 & 0.56 \\
\hline Yulania puberula & 104126 & 0.78 & 2038 & 0.56 \\
\hline Yulania shizhenii & 104103 & 0.78 & 2035 & 0.56 \\
\hline Yulania denudata_JN867577.1 & 103967 & 0.78 & 2041 & 0.56 \\
\hline Yulania kobus_NC̄023237.1 & 103946 & 0.78 & 2040 & 0.56 \\
\hline Yulania liliiflora_JX280397.1 & 103412 & 0.78 & 2054 & 0.57 \\
\hline
\end{tabular}

\section{Evolutionary System of Magnoliaceae Juss}

The taxonomical system of Magnoliaceae Juss. has always been more controversial. Dandy system [11] had the biggest impact, which included 12 genera: Manglietia Blum., Magnolia L., Talauma Juss., Alcimandra Dandy, Aromadendron Blum., Pachylarnax Dandy, Kmeria (Pierre) Dandy, Elmerrrillia Dandy, Michelia L., Paramichelia Hu, Tsoongiodendron Chun and Liriodendron L.. Based on the system, Y. H. Law system [12] increased 3 genera: Manglietiastrum Law, Dugandiodendron G. Lozano-Contreras, Parakmeria $\mathrm{Hu}$ et Cheng. N. H. Xia system [13] increased 5 genera: Houpoea N. H. Xia \& C. Y. Wu, Oyama (Nakai) N. H. Xia \& C. Y. Wu, Lirianthe Spach, Woonyoungia Law, Yulania Spach. Most of these genera were not supported by the phylogeny. Bailon system [14] and Nooteboom system [15] only included 2 genera: Magnolia L. and Liriodendron L., which were adopted by the NCBI database.

Traditional taxonomical and phylogenic system sometimes are inevitably partial and subjective [1]. To establish a scientific system, the PHS and EVS are analyzed based on the chloroplast complete genome of 28 species of Magnoliaceae using the types of 4 species respectively: Yulania denudata (Desr.) D. L. Fu, Magnolia fordiana (Oliver) $\mathrm{Hu}$, Michelia odora (Chun) Nooteboom \& B. L. Chen, Liriodendron tulipifera L., and the results are shown in Table 8 to 11 .

It can be seen from Table 8 to 11 that using the types of Yulania denudata (Desr.) D. L. Fu, Magnolia fordiana (Oliver) $\mathrm{Hu}$, Michelia odora (Chun) Nooteboom \& B. L. Chen and Liriodendron tulipifera L. respectively, Magnoliaceae Juss. obviously include 4 natural genera: Yulania Spach, Magnolia L., Michelia L. and Liriodendron L., which all have the same boundaries: $\quad$ PHS(17bp) $\geq 0.93$ (inner genus) and PHS $(17 b p)<0.93$ (inter genera), and EVS $(17 b p) \geq 0.83$ (inner genus) and $\operatorname{EVS}(17 \mathrm{bp})<0.83$ (inter genera, except for Magnolia sinica (Y. W. Law) Nooteboom). For PHS being more easily calculated just need two samples, so 
$\operatorname{PHS}(17 \mathrm{bp})=0.93$ can be regarded as the generic boundary of Magnoliaceae. Based on the boundary, it can be concluded that Manglietia Blum., Pachylarnax Dandy, Manglietiastrum Law, Parakmeria Hu et Cheng, Houpoea N. H. Xia \& C. Y. $\mathrm{Wu}$, Woonyoungia Law, Alcimandra Dandy, and Tsoongiodendron Chun are not supported by chloroplast complete genomic evolutionomy. By means of morphological evolutionomy, it can be basically concluded that Talauma Juss., Kmeria (Pierre) Dandy, Aromadendron Blum., Elmerrrillia Dandy, Dugandiodendron G. Lozano-Contreras, Oyama (Nakai) N. H. Xia \& C. Y. Wu, Lirianthe Spach and Paramichelia $\mathrm{Hu}$ are not supported too.

According the evolutionary continuity principle, based on PHS and EVS of 28 species, the evolutionary system of Magnoliaceae Juss. can be established as Figure 2, which scientifically overcomes the partiality and subjectivity of traditional and phylogenic system.

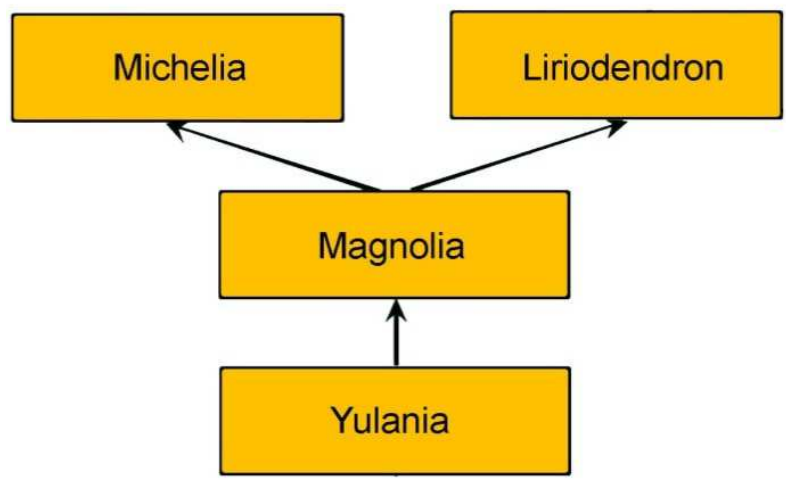

Figure 2. Evolutionary system of genera of Magnoliaceae Juss. based on the chloroplast genomic and morphological evolutionomy.

The genera of Magnolia L., Michelia L. and Liriodendron L. of Magnoliaceae Juss. were widely accepted by most of taxonomical system [2-8, 11-13, 15-19, 21-23], but the genus of Yulania Spach had been regarded as Magnolia L. by most botanical authorities [16-18]. As Yulania Spach was scientifically confirmed in 2001 [19], its species resources were also gradually identified [20-23].

\section{Evolutionary Taxa of Yulania sect. Yulania Subsect. Cylindricae}

\subsection{Yulania Subsect. Cylindricae (Spongb.) D. L. Fu, Subsect. Comb. Nov., Figures 3 to 5}

Magnolia sect. Cylindricae S. A. Spongberg, in D. R. Hunt (ed.), Magnolias \& their allies: 115. 1998. Yulania sect. Cylindrica (Spongb.) T. B. Zhao \& Z. X. Chen, nom. illegit. (laps. cit.) in T. B. Zhao et al., Shijie Yulanshu Zhiwu Ziyuan yu Zaipei Liyong. 175.

Deciduous trees. Hornotini-branchlets reddish purple, often hairy. Leaves obovate, rarely elliptic, surface green, glabrous or sparsely puberulent, reticulate veins flat or depressed and rimous when dried, lower surfaces pallid green or glaucescent densely puberulent. Flower buds terminal, bracts membranous, bractoid or peruloid. Flower precocious, often sepals 3 and tepals 6. Fruit aggregates cylindric, often curved or not.

Typus: Yulania cylindrica (Wils.) D. L. Fu, J. Wuhan Bot. Res., 19(3): 198. 2001; Magnolia cylindrica Wils. in J. Arnold Arbor. 8: 109. 1927.

Three species could be confirmed in this subsection: Yulania puberula D. L. Fu, sp. nov., Yulania cylindrica (Wils.) D. L. Fu, Yulania shizhenii D. L. Fu et F. W. Li, mainly distributed in Hubei, Anhui, Sichuan and other provinces of South China.

The species of this subsection are the most primitive taxa of fruit plants, and plays an important role in the study of origin and evolution of fruit plants.

\subsection{Yulania Puberula D. L. Fu, sp. Nov., Figure 3}

Arbor decidua. Ramuli crassi, lenticellis rarioribus rotundis leviter protuberantibus, hornotini purpureo-rubri pubescentes et puberuli nitidi, ramuli abbreviati 3 5-nodi, internodiis ca. 3 $\mathrm{mm}$ longis leviter crassis pallide brunneis sparse puberulis. Gammae foliiferae conoideae vel ovoideo-conoideae 0.5 1.2 $\mathrm{cm}$ longae et $0.2 \sim 0.4 \mathrm{~cm}$ diam. dense pallide adpresso-flavo-pubescentes. Alabastra solitaria, terminalia, ovoidea circa $2.0 \mathrm{~cm}$ longa et $1.0 \mathrm{~cm}$ diam. extus dense pallide adpresso-flavo-villosa. Folia alterna tenuiter coriacea elliptica late oblanceolata vel oblanceolato-elliptica $6.5 \sim 10.5 \mathrm{~cm}$ longa et $2.0 \sim 3.5 \mathrm{~cm}$ lata apice acuminata vel acuta basi cuneata vel late cuneata margine integra supra viridia parte facile lateritoscentia in sicco glabra nitida nervis et nervulis impressis rimosis in sicco subtus pallide viridia, dense puberula, pilis $1.5 \sim 2.5 \mathrm{dmm}$ longis, costis manifeste prominentibus dense puberulis, pilis usque ad $5.0 \mathrm{dmm}$ longis, nervis lateralibus 10 15-jugis; petioli graciles $0.8 \sim 2.5 \mathrm{~cm}$ longi, dense puberuli, cicatriculis stipularum $2 \sim 3 \mathrm{~mm}$ longis interdum longitudine attingentibus $1 / 3$ petiolorum partem aequantibus. Flores ante folia aperti, albi; pedicelli flavo-viriduli $0.8 \sim 1.2 \mathrm{~cm}$ longi et $0.7 \sim 0.8$ diam. dense albi pubescentes vel villosi; bractae 1, peruli-morphis; sepala $(1 \sim) 3$ alba carnosa linearia, petala $6(\sim 8)$ alba carnosa spathulata vel spathulato-obovata, $7.0 \sim 10.0 \mathrm{~cm}$ longa et $3.5 \sim 6.0 \mathrm{~cm}$ lata extus costis roseolis basi vivido-purpureo-rubra. Stamina numerosa ca. 65 in quequo flore, $0.8 \sim 1.1 \mathrm{~cm}$ longa, pallide purpureo-rubra extus costis roseolis, thecis lateribus longitudinali-dehiscentibus, connetivis apice cum mucronibus triangulis ca. $1.5 \mathrm{~mm}$ longis; filamentis ca. $2.5 \mathrm{~mm}$ longis rectangularibus pallide purpureo-rubris; gynoecium subcylindricumca. $1.6 \mathrm{~cm}$ longum, pistilla simplicia numerosis ca. $40 \sim 45$ in quequo flore, glabra, ovariis pallide flavo-viridulis interdum pallide purpureo-rubris fasciatis protuberantis costis purpureo-rubris, stylis et stigmatibus extus pallide purpureo-rubris. Folliceta breviter cylindrica purpureo-rubra ca. $5.0 \mathrm{~cm}$ longa et $2.8 \mathrm{~cm}$ diam. saepe curvata; semina depresso-globosa $0.7 \mathrm{~mm}$ longa et $0.7 \mathrm{~cm}$ lata.

Hubei: D. L. Fu2018032601 (Holotypus, hic designatus, CAF) branchlets with flower, were collected from Mountain Wudang at alt. about $970 \mathrm{~m}$ on Mar. 26, 2018, by D. L. Fu, and D. L. Fu 2017100801 (Paratypus, CAF), branchlets with fruit, 
flower buds and leaves were collected from the same position on Oct. 08, 2017.

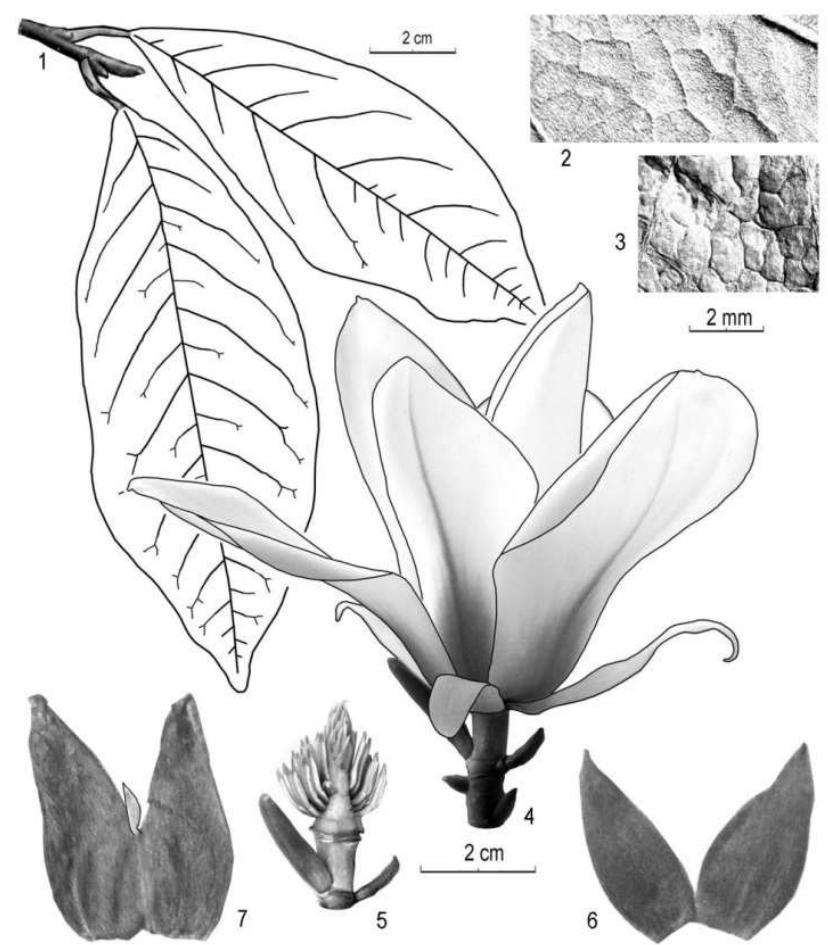

Figure 3. Yulania puberula D. L. Fu, sp. nov. 1. branchlet with leaves; 2. lower surface of leaf (to show densely alba puberulent); 3. upper surface of leaf (reticulate veins depressed and rimous when dried); 4. flower; 5. flower with tepals removed to show gynoecium and androecium; 6. perule; 7. bract (peruloid). (drawn by D. L. Fu)

The chloroplast complete genome of this new species is mostly similar to Y. cylindrica (Wils.) D. L. Fu and PHS = 0.992 .

The main typici-evolutionary characters including diagnostic differences and particularities of the new species are that the hornotini-branchlets reddish purple, leaves thin corious, elliptic or oblanceolati-elliptic, reticulate veins depressed and rimous when dried, lower surfaces densely puberulent; Flower precocious, sepals 3 and tepals 6 , bract 1 , peruloid. That the leaves oblanceolati-elliptic, reticulate veins depressed and rimous when dried, bract peruloid are also the new typci-evolutionary characters of the species, which can be used to distinguish to similar species Yulania cylindrica (Wils.) D. L. Fu [16-23], Y. shizhenii D. L. Fu et W. F. Li [20] and other species of the genus. Furthermore the leaf reticulate veins depressed and rimous when dried and bract peruloid are also the most primitive morphological signs in fruit plants.

Distribution: The species is mainly distributed in Hubei province of China.

The species is the most primitive fruit plants and will play an important role in researching the Evolutionomy of Magnoliaceae Juss. even that of Fructophyta D. L. Fu\& H. Fu, but it is very rare in population, so the authors intensively proposed that the species should be protected as first level protection.

\subsection{Yulania Shizhenii D. L. Fu et F. W. Li, sp. Emend., Figure 4}

Bull. Bot. Res. (Haerbin), 30 (4): 387-389. 2010, p.p. excl. paratypus; T. B. Zhao et al., Shijie Yulanshu Zhiwu Ziyuan yu Zaipei Liyong: 258-259. 2013. —-Yulania kobus var. shizhenii T. B. Zhao et Z. X. Chen, in Shijie Yulanshu Zhiwu Zhongzhi Ziyuan Zhi: 67-68. 2013. —Yulania liliiflora var. shizhenii T. B. Zhao et Z. X. Chen, in Henan Yulan Zaipei: 226-227. 2015.

Characteres specifici peculiares prioritatum et evolutionales typici: Ramuli graciles cum limbo-cicatricibus petiolorum hornotini purpureo-rubri. Folia longe obovata membranacea subtus dense puberula, petiolis base dilatatis. Flores ante folia aperti; sepala 3 et tepala 6; stamina alba, filamentis latiora quam antheris.

The main typici-evolutionary characters: the branchlets slender with eave scars of petioles, hornotini-branchlets reddish-purple; leaves long obovate, membranous and the lower surfaces densely puberulent; petiole bases enlargement; Flower precocious, sepals 3 and tepals 6, stamens white and filaments wider than anthers.

Typus: D. L. Fu 200103161 (Holotypus, CAF), D. L. Fu 2017110501 (Epitypus, hic designatus, CAF). The types were collected from Chengdu city of Sichuan province of China and kept at Chinese Academy of Forestry (CAF).

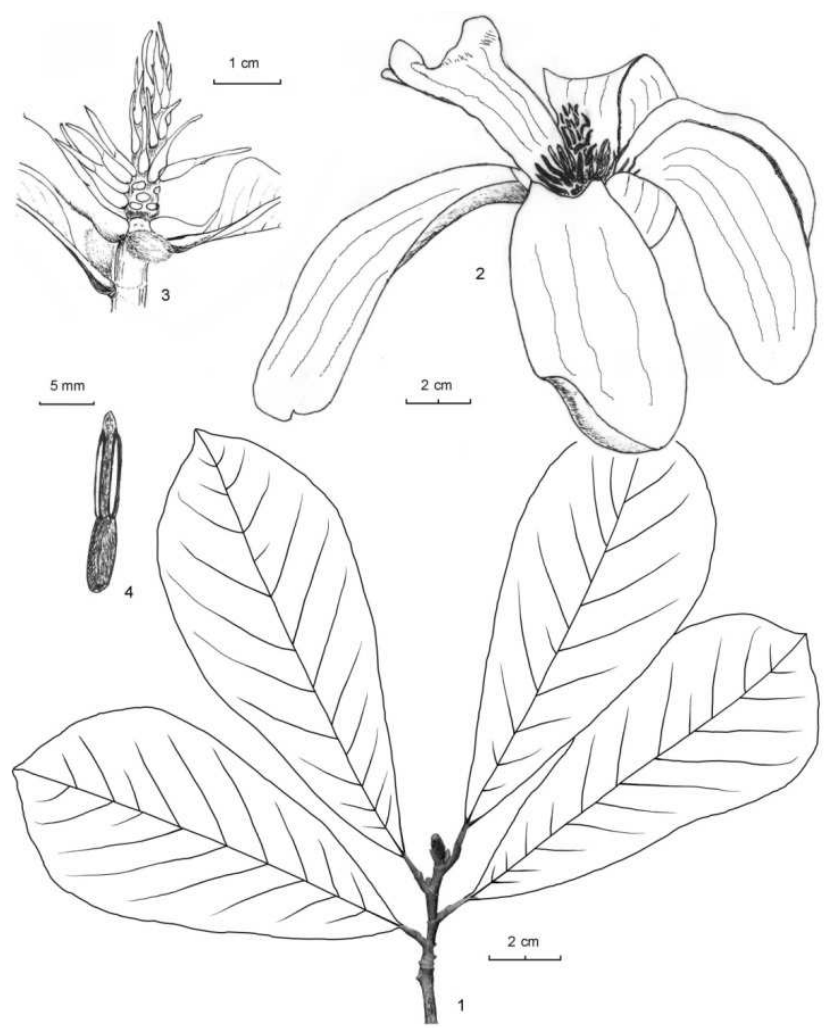

Figure 4. Yulania shizhenii D. L. Fu et F. W. Li, sp. emend. 1. branchlet with leaves (drawn by H. Fu); 2. flower; 3. gynoecium and androecium; 4. stamen. (from Bull. Bot. Res.)

This species is named for commemorating Li Shizhen, a great pharmacologist and botanist of ancient China.

Distribution: The species is distributed in Sichuan province 
of China.

The species is an excellent ornamental tree species with the pure white flowers, open in early spring. Its flower buds into Chinese medicine, called Xinyi. As one of the most primitive taxa, it should be recommended for inclusion in the national first-level list of protected plants for its small number of populations and very important role in the origin and evolution of fruit plants.

\subsection{Yulania Cylindrica (Wils.) D. L. Fu, Figure 5}

J. Wuhan Bot. Res., 19(3): 198. 2001; Xia Nianhe et al., Flora of China vol. 7: 75. 2008; _-Magnolia cylindrica Wils. in J. Arnold Arbor. 8: 109. 1927. Typus: R. C. Ching 2949 (A, collected from Mountain Huang in Anhui province of China).

Yulania fragarigynandria T. B. Zhao, Z. X. Chen et H. T. Dai, in T. B. Zhao et al. Shijie Yulanshu Zhiwu Ziyuan yu Zaipei Liyong: 236-237. 2013. Type: 200504041(HEAC); T. B. Zhao et al. Shijie Yulanshu Zhiwu Zhongzhi Ziyuan Zhi: 78-79. 2013; T. B. Zhao et al., Henan Yulan Zaipei: 270-271. 2015.

Yulania gynophora T. B. Zhao, Z. X. Chen et J. Zhao, inT. B. Zhao et al. Shijie Yulanshu Zhiwu Ziyuan yu Zaipei Liyong: 288-289. 2013. Type: 200203131(HEAC); T. B. Zhao et al. Shijie Yulanshu Zhiwu Zhongzhi Ziyuan Zhi: 79-80. 2013; T. B. Zhao et al., Henan Yulan Zaipei: 269-270. 2015.

Yulania varians T. B. Zhao, Z. X. Chen et Z. F. Ren, in T. B. Zhao et al. Shijie Yulanshu Zhiwu Ziyuan yu Zaipei Liyong: 289-291. 2013. Type: 201303222(HEAC); in T. B. Zhao et al. Shijie Yulanshu Zhiwu Zhongzhi Ziyuan Zhi: 82-83. 2013; in T. B. Zhao et al., Henan Yulan Zaipei: 272-273. 2015.

Yulania anhueiensis T. B. Zhao, Z. X. Chen et J. Zhao, in T. B. Zhao et al. Shijie Yulanshu Zhiwu Ziyuan yu Zaipei Liyong: 302-303. 2013. Type: 200503301(HEAC); in T. B. Zhao et al. Shijie Yulanshu Zhiwu Zhongzhi Ziyuan Zhi: 78-79. 2013; in T. B. Zhao et al., Henan Yulan Zaipei: 268-269. 2015.

Magnolia glabrata auct. Non Law et R. Z. Zhou ined.: Y.

H. Liu, Magnolias of China: pict.(top-left, p.61). 2004.

Yulania axilliflora auct. non(T. B. Zhao et al.) D. L. Fu: T. B. Zhao et al., Henan Yulan Zaipei: t.74(6). 2015.

The main typici-evolutionary characters: Fruit aggregates cylindric, often short and thick, and not curved, the follicles dehiscing by dorsal suture or not, but the apical portions falling away individually or in irregular masses leaving the seeds suspended on persistent central axes [17]. It can be clearly distinguished from other species of Yulania.

The species was described and illustrated as some new species such as Yulania fragarigynandria T. B. Zhao, Z. X. Chen et H. T. Dai [21-23], Yulania gynophora T. B. Zhao, Z. X. Chen et J. Zhao [21-23], Yulania varians T. B. Zhao, Z. X. Chen et Z. F. Ren [21-23] and Yulania anhueiensis T. B. Zhao, Z. X. Chen et J. Zhao [21-23], which all were synonyms of Yulania cylindrica (Wils.) D. L. Fu.

Distribution: The species is distributed in Anhui, Zhejiang, Jiangxi, Fujian, Hubei and Henan province of China, in broad-leaved forest at an altitude of $600 \sim 1600 \mathrm{~m}$.

The species with white or whitish-purple flowers and red fruits, very beautiful, is an excellent ornamental tree species in urban and rural areas; Flower buds is used as Chinese medicine for "Xinyi", and is also an excellent spice plant resource.

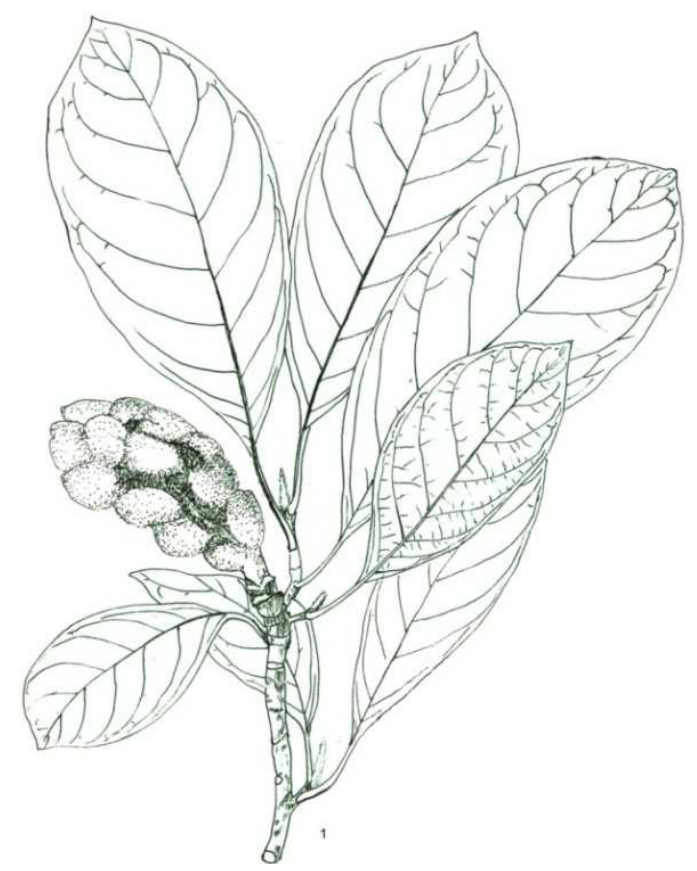

Figure 5. Yulania cylindrica (Wils.) D. L. Fu. 1. branchlet with leaves and fruit. (from Rare and Endangered Plants in China).

\section{Conclusion}

Scientifically overcoming the limitation of Phylogeny sometimes being partial and subjective, typical algorithm for the genomic evolutionomy, is a scientifically new method and new tool to solve the puzzle of evolutionomy of fruit plants. Based on the results of chloroplast genomic analyses, it can be concluded that Fructophyta are originated from Ginkgoopsida of Gymnospermophyta, not from Cycadopsida thought by the euanthium-theory or Chlamydopsermopsida thought by the pseudoanthium-theory, which will create a new situation in the development of evolutionomy of Fructophyta D. L. Fu \& H. Fu.

Among some representative species of Fructophyta, Ginkgo biloba has the closest relatively evolutionary relationship with Yulania puberula indicating that the new species is the relatively most primitive species of fruit plants. It is consistent with the research results of morphological evolutionomy, but it is not consistent with the current people's cognition. Therefore, it will certainly promote the research of re-understanding of the evolutionomy of fruit plants and promote the development of the evolutionomy of Fructophyta D. L. Fu \& H. Fu.

Based on the results of evolutionomic analyses of chloroplast genomes and morphology, the evolutionary system of Magnoliaceae includes 4 natural genera: Yulania Spach, Magnolia L., Michelia L. and Liriodendron L., whose boundaries all are $\mathrm{PHS}(17 \mathrm{bp})=0.93$ (chloroplast complete genomes). This will create a new situation in the scientific classification of Magnoliaceae Juss. and will also promote the development of the evolutionomy of Fructophyta D. L. Fu \& H. Fu. 


\section{Acknowledgements}

This work was supported by the Fundamental Research Funds for the Central Non-profit Research Institution of CAF (CAFYBB2016MA009). The chloroplast genomes of three species of Yulania subsect. Cylindricae were assembled and annotated by Huitong Biotech Co., Ltd. We are grateful to Prof. Wencai Wang, plant taxonomist and academician of the Chinese Academy of Sciences, and Prof. Yingping Wang of CSIRO (Commonwealth Scientific and Industrial Research Organization) for their valuable suggestions and corrections in some writing text, and thank Engineers Zhengliu Wei et al. of Forest Bureau of Mountain Wudang Tourism Economic Zone, Weihua Fang et al. of Huangshan Arboretum of Anhui Province for their helps in investigating of the plant resources.

\section{References}

[1] D. L. Fu and H. Fu. "An evolutionary continuity principle for evolutionary system of organism divisions". American Journal of Agriculture and Forestry, vol. 6, no. 3, pp. 60-64, 2018. DOI: 10.11648/j.ajaf.20180603.14.

[2] H. D. Zhang, Y. H. Huang, R. H. Miao, C. X. Ye, W. B. Liao, and J. H. Jin. "Systematics of Spermatophyta". Beijing: Science Press. 72-626, 2004. [in Chinese]

[3] J. W. Wang. "Taxonomy of Spermophyta". Beijing: Higher Education Press. 290-295, 1985. [in Chinese]

[4] J. Hutchinson. "The families of flowering plants". Oxford: Oxford University Press. 1959.

[5] A. L. Takhtajan. "Outline of the classification of flowering plants (Magnoliophyta)". Bot. Rev. vol. 46 no. 3. pp. 227-359. 1980.

[6] A. Cronquist. "An integrated system of classification of flowering plants". New York: Columbia University Press, 1-1262, 1981.

[7] Z. Y. Wu, Y. C. Tang, A. M. Lu, Z. R. Chen, and D. Z. Li. "Synopsis of a new 'polyphyletic-polychronic-polytopic' system of the angiosperms". Acta Phytotaxonomica Sinica, vol. 40, no. 4, pp. 289-322, 2002.

[8] D. Z. Li (translator). "Plant systematic, A phylogenetic approach”. Beijing: Higher Education Press. 001-496, 2012. [in Chinese]

[9] N. Li., W. Huang, Q. Shi, Y. Zhang, and L. Song. "A CTAB-assisted hydrothermal synthesis of VO2(B) nanostructures for lithium-ion battery application". Ceram. Int. vol. 39, pp. 6199-6206, 2013. DOI: 10.1016/j.ceramint.2013.01.039.
[10] M. Lohse, O. Drechsel, and R. Bock. "Organellar Genome DRAW (OGDRAW): A tool for the easy generation of high-quality custom graphical maps of plastid and mitochondrial genomes". Curr. Genet. vol. 52, pp. 267-274, 2007.

[11] J. E. Dandy. Magnoliaceae. In: Hutchinson J (ed.). "The Genera of Flowering Plants, Dicotyledons", Oxford, 50-57, 1964.

[12] Y. H. Liu. "A preliminary study on the taxonomy of the family Magnoliaceae". Acta Phytotaxonomica Sinica, vol. 22, pp. 89-109, 1984.

[13] N. H. Xia. "A New classification System of the Family Magnoliaceae”. In: Xia N H, Zheng Q W, Xu F X, Wu Q G. Proceedings of the Second International Symposium on the Family Magnoliaceae. Wuhan: Huazhong University of Science \& Technology Press. 12-38, 2009.

[14] H. Baillon. "Memoire sur la famille des Magnoliaceae". Adansonia vol. 7, pp. 1-16, 1866.

[15] N. H. Xia, Y. H. Liu, H. P. Nooteboom. Magnoliaceae. In: Wu Z Y, P. H. Raven, Hong D Y. "Flora of China", Beijing: Science Press \& St. Louis, MO: Missouri Botanical Garden Press. vol. 7, pp. 71-77, 2008.

[16] D. J. Callaway. “The World of Magnolias". Portland: Timer Press. 135-174, 1994.

[17] D. Hunt. "Magnolias and their allies". Sherborne: International Dendrology Society and The Magnolia Society. 104-126, 1998.

[18] Y. H. Liu. "Magnolias of China". Beijing: Science Press, 44-55, 2004.

[19] D. L. Fu. "Notes on Yulania Spach". Journal of Wuhan Botanical Research, vol. 19, no. 3, pp. 191-198, 2001.

[20] D. L. Fu, D. L. Zhang, F. W. Li, J. H. Sun, and J. H. Ren. "Two New Species of Yulania Spach from Sichuan Province of China”. Bulletin of Botanical Research, vol. 30, no. 4, pp. 385-389, 2010.

[21] T. B. Zhao, G. H. Tian, D. L. Fu, and D. X. Zhao. "Shijie Yulanshu Zhiwu Ziyuan yu Zaipei Liyong”. Beijing: Science Press, 179-383, 2013. [in Chinese]

[22] T. B. Zhao, Z. F. Ren, and G. H. Tian. "Shijie Yulanshu Zhiwu Zhongzhi Ziyuan Zhi”. Zhengzhou: Yellow River Conservancy Press, 63-64, 2013. [in Chinese]

[23] T. B. Zhao, L. H. Song, G. H. Tian, and Z. X. Chen. "Henan Yulan Zaipei". Zhengzhou: Yellow River Conservancy Press, 157 - 161, 2015. [in Chinese] 